\title{
FLUTUAÇÃO POPULACIONAL E DISTRIBUIÇÃO VERTICAL DE BREVICORYNE BRASSICAE (L.) (HEMIPTERA: APHIDIDAE) EM COUVE ${ }^{(1)}$
}

\author{
FRANCISCO J. CIVIDANES ${ }^{(2,4)} ;$ DURVALINA M. MATHIAS DOS SANTOS $^{(3)}$
}

\begin{abstract}
RESUMO
O conhecimento da dinâmica populacional e da distribuição vertical de insetos pragas em plantas hospedeiras é fundamental para o desenvolvimento de programas de manejo integrado de pragas. No presente trabalho efetuou-se um levantamento populacional de formas ápteras do pulgão Brevicoryne brassicae (L.), visando determinar a época de maior densidade populacional e a distribuição vertical em plantas de couve, (Brassica oleracea L. var. acephala DC.), cultivadas em Jaboticabal, SP. O estudo foi realizado durante as safras de brássicas de 1998 e 1999, efetuando-se correção da acidez do solo por meio de aplicação de calcário apenas no campo utilizado em 1998. A amostragem dos pulgões foi feita visualmente em folhas classificadas em três categorias: apical, mediana e basal. Nas duas safras estudadas, a infestação de $B$. brassicae na couve atingiu a maior densidade populacional em setembro, diminuindo rapidamente a seguir. Nos dois campos não se observou a mesma distribuição de $B$. brassicae em folhas apicais, medianas e basais. Os fatores que podem ter contribuído para as diferenças observadas no padrão de distribuição do pulgão devem estar relacionados com a precipitação pluvial e o calcário magnesiano.
\end{abstract}

Palavras-chave: Insecta, pulgão, calcário, Brassica oleracea.

\author{
ABSTRACT \\ POPULATIONAL FLUCTUATION AND VERTICAL (WITHIN-PLANT) \\ DISTRIBUTION OF BREVICORYNE BRASSICAE (L.) \\ (HEMIPTERA: APHIDIDAE) ON KALE
}

The knowledge of the population dynamics and vertical distribution of insect pests in host plants are fundamental for the development of programs of integrated pest management. In the present work, a population survey of apterous cabbage aphid, Brevicoryne brassicae (L.), was carried out aiming to determine the period when this aphid reachs the highest population density and the within-plant distributions on its common host, kale, Brassica oleracea L. var. acephala DC., cultivated in Jaboticabal, State of São Paulo. The study took place during the 1998 and 1999 Brassica seasons, and limestone treatment was only made in the field cultivated in 1998. The cabbage aphid was sampled by visual search on leaves classified in three categories: top, middle and basal. In the two studied seasons, the B. brassicae infestations reached the highest population density in September, decreasing quickly afterwards. In the two fields, the same distributions of $B$. brassicae was not observed on top, middle and basal leaves. The factors that might have contributed to the differences observed in that distribution pattern were rainfall and magnesium limestone.

Key words: Insecta, cabbage aphid, limestone, Brassica oleracea.

(1) Recebido para publicação em 29 de janeiro e aceito em 11 de dezembro de 2002.

$\left(^{2}\right)$ Departamento de Fitossanidade, FCAV/UNESP, Via de Acesso Prof. Paulo D. Castellane s/n. ${ }^{\circ}$, $14870-000$ Jaboticabal (SP). E-mail: fjcivida@fcav.unesp.br.

$\left(^{3}\right)$ Departamento de Biologia Aplicada à Agropecuária, FCAV/UNESP.

$\left({ }^{4}\right)$ Com bolsa de produtividade científica do CNPq. 


\section{INTRODUÇÃO}

O pulgão-da-couve, Brevicoryne brassicae (L.), tem sido considerado uma importante praga associada com Brassica spp., encontrando-se amplamente distribuído nas regiões temperadas e subtropicais do mundo (ElLIS e Singh, 1993). Em nosso país é considerada praga-chave da cultura da couve (SALGADO, 1983).

As informações sobre a distribuição vertical de insetos-praga na planta hospedeira são fundamentais para o desenvolvimento de planos de amostragens (TRICHILO et al., 1993), reduzindo o tempo e custos necessários para o monitoramento da praga, sem que haja diminuição da confiabilidade dos resultados (WILSON et al., 1982). Aparentemente, $B$. brassicae prefere as folhas mais jovens de espécies de brássicas (VAn EMden e Bashford, 1971; Trumble, 1982; Ceron-Hernandez e Salguero, 1995). Todavia, a distribuição vertical dessa espécie pode mudar com a idade da planta hospedeira (CHURCH e STRICKLAND, 1954; GEORGE, 1957).

O conhecimento da flutuação populacional de um inseto praga é necessário para o desenvolvimento de programas de manejo integrado de pragas (Wright e CONE, 1988). De acordo com considerações feitas por Wellings e Dixon (1987), as populações de pulgões podem flutuar e manter níveis altos de densidade ou, em alguns casos, períodos de abundância são seguidos por períodos de baixa densidade.

A abundância de pulgões mostra-se altamente sazonal, podendo variar consideravelmente de um ano para outro. Além disso, os padrões de flutuação das populações de uma determinada espécie podem diferir entre regiões geográficas distintas, entre populações que se desenvolvem na mesma região por vários anos e entre as populações vizinhas que se desenvolvem ao mesmo tempo.

As alterações que ocorrem nas densidades populacionais de pulgões são pouco entendidas; no Brasil os estudos sobre a dinâmica populacional de B. brassicae são escassos, e as informações disponíveis relacionam-se com a ação de inimigos naturais (PEReIra e SMith, 1976; Souza e Bueno, 1992; Bueno e Souza, 1993) e com os efeitos dos fatores meteorológicos sobre as formas aladas (OLIVEIRA, 1971; Rossi et al., 1990).

O objetivo do presente trabalho foi efetuar um levantamento populacional de formas ápteras de $B$. brassicae, visando determinar a distribuição vertical e a época de maior densidade populacional em plantas de couve, quando cultivada na região de Jaboticabal, SP.

\section{MATERIAL E MÉTODOS}

O estudo foi desenvolvido em dois campos de couve (Brassica oleracea L. var. acephala DC.), híbrido Agroceres HE-350, situados na área experimental dos Departamentos de Produção Vegetal (campo 1) e Fitossanidade (campo 2), da Faculdade de Ciências Agrárias e Veterinárias (FCAV), Universidade Estadual Paulista (UNESP), Campus de Jaboticabal, de abril de 1998 a dezembro de 1999.

O campo 1 apresentava área de $1.300 \mathrm{~m}^{2}$ (20 x $65 \mathrm{~m}$ ) de Latossolo Vermelho Eutroférrico. As mudas de couve foram transplantadas em 10/3/98, sendo cultivadas, aproximadamente, 2.300 plantas em espaçamento $1,0 \times 0,5 \mathrm{~m}$. Durante o experimento, novas mudas foram introduzidas, repondo aquelas que pereceram principalmente devido ao ataque de formigas cortadeiras. As datas desses transplantes foram: 20/7/98, 07/10/98, 27/10/98, 30/12/98 e 12/3/99.

No campo 2, com área de $520 \mathrm{~m}^{2}(8 \times 65 \mathrm{~m})$ de Latossolo Vermelho, foram cultivadas cerca de 530 plantas em espaçamento $1,0 \times 1,0 \mathrm{~m}$. As mudas foram transplantadas em $10 / 6 / 99$, não sendo introduzidas novas mudas posteriormente.

As análises químicas do solo, campo 1 e campo 2, foram realizadas no Laboratório de Análise de Solo e Planta do Departamento de Solos e Adubos da FCAV/UNESP (Quadro 1). Para o campo 1 foi feito tratamento de calagem e adubação, enquanto no campo 2 utilizou-se apenas adubação.

A análise de solo do campo 2 indicou $\mathrm{pH}=$ 5,5 , considerado adequado para o cultivo da cultura de couve, pois segundo FilgueIRA (2000), essa cultura pode ser cultivada em solos que apresentem $\mathrm{pH}$ entre 5,5 e 6,5 .

Para a calagem no campo 1 foi utilizado 2,8 t.ha ${ }^{-1}$ de calcário magnesiano com PN $=98 \%$, PRNT $=90 \%, \mathrm{CaO}=36 \%$ e $\mathrm{MgO}=9 \%$, aplicado em área total e incorporado 20 dias antes do plantio.

Em ambos os campos, para a adubação de plantio foram utilizados $200 \mathrm{~kg} \cdot \mathrm{ha}^{-1}$ de sulfato de amônio, $100 \mathrm{~kg} \cdot \mathrm{ha}^{-1}$ de cloreto de potássio e $2 \mathrm{~kg} \cdot \mathrm{ha}^{-1}$ de ácido bórico, além de $900 \mathrm{~kg} \cdot \mathrm{ha}^{-1}$ e $2.000 \mathrm{~kg} \cdot \mathrm{ha}^{-1}$ de superfosfato simples, respectivamente, para o campo 1 e 2 . A adubação de cobertura foi realizada entre 30 a 45 dias, usando-se $200 \mathrm{~kg} \cdot \mathrm{ha}^{-1}$ de sulfato de amônio e $30 \mathrm{~kg} \cdot \mathrm{ha}^{-1}$ de cloreto de potássio para o campo 1, e $195 \mathrm{~kg} \cdot \mathrm{ha}^{-1}$ de sulfato de amônio e $43 \mathrm{~kg} \cdot \mathrm{ha}^{-1}$ de cloreto de potássio, para o campo 2 .

Nos dois campos estudados, foram realizadas capinas periódicas para manter a cultura da couve no limpo. A irrigação foi feita por sulco (campo 1) e por gotejamento (campo 2), duas vezes por semana. 
Quadro 1. Propriedades químicas do solo nos dois campos estudados. Jaboticabal (SP), 1998/99

\begin{tabular}{|c|c|c|c|c|c|c|c|c|c|c|}
\hline Local & $\mathrm{PH}^{(1)}$ & M.O. & $\mathrm{P}^{(2)}$ & $\mathrm{K}$ & $\mathrm{Ca}$ & $\mathrm{Mg}$ & $(\mathrm{H}+\mathrm{Al})$ & SB & $\mathrm{T}$ & $\mathrm{V}$ \\
\hline & & g.dm ${ }^{-3}$ & $\mathrm{mg} \cdot \mathrm{dm}^{-3}$ & \multicolumn{6}{|c|}{$\mathrm{mmol}_{\mathrm{c}} \cdot \mathrm{dm}^{-3}$} & $\%$ \\
\hline Campo 1 & 5,1 & 29 & 147 & 10,8 & 40 & 12 & 47 & 62,8 & 109,8 & 57 \\
\hline Campo 2 & 5,5 & 26 & 16 & 6,3 & 33 & 12 & 38 & 51,3 & 89,3 & 57 \\
\hline
\end{tabular}

( ${ }^{1} \mathrm{Cacl}_{2} \cdot\left({ }^{2}\right)$ Resina.

Ressalta-se que, por problemas técnicos, no campo 1 em agosto e setembro de 1998, a irrigação foi feita por aspersão.

No campo 1, efetuou-se o levantamento populacional de formas ápteras de B. brassicae, de abril de 1998 a julho de 1999, em intervalos de 7 a 10 dias; no campo 2, foi de junho a dezembro de 1999, em intervalos de aproximadamente 10 dias. Os pulgões foram amostrados em três folhas por planta de couve, perfazendo um total de 20 plantas escolhidas ao acaso no campo, com as folhas sendo agrupadas eqüitativamente em três categorias: a) apical: folha jovem e não totalmente expandida; $b$ ) mediana: folha adulta e totalmente expandida; c) basal: folha senescente e com visível amarelecimento.

A contagem do número de pulgões foi realizada utilizando como critério a superfície foliar onde ocorria a maior colônia de adultos e ninfas, situada nos limites da área circular de um vazador de metal de $3,5 \mathrm{~cm}$ de diâmetro, considerada como unidade amostral (área $=9,62 \mathrm{~cm}^{2}$ ), conforme Sousa (1990).

Verificaram-se as diferenças entre as ocorrências de B. brassicae nas três categorias de folhas, considerando a média dos dados obtidos nas diferentes datas de amostragens efetuadas em cada mês do levantamento populacional. Para se processar as análises, os dados foram transformados em $\log (x+1)$ e submetidos à análise da variância, sendo as médias comparadas pelo teste de Tukey.

A influência de fatores meteorológicos foi avaliada pela análise de correlação simples, sendo considerados os fatores: temperatura máxima, mínima e média $\left({ }^{\circ} \mathrm{C}\right)$, umidade relativa $(\%)$, insolação (horas) e precipitação pluvial $(\mathrm{mm})$, obtidos junto à Estação Agroclimatológica da FCAV/UNESP. Os pulgões amostrados foram representados pelo número médio dos indivíduos observados nas três categorias de folhas de couve.

Para os fatores meteorológicos de temperatura, umidade relativa e insolação utilizaram-se as médias; para a precipitação pluvial foram consideradas a soma (registradas nos períodos de sete dias anteriores) e as datas de amostragem de B. brassicae.

\section{RESULTADOS E DISCUSSÃO}

O levantamento populacional de formas ápteras de B. brassicae nos campos 1 e 2 (Quadros 2 e 3 , respectivamente) mostrou que a maior densidade de pulgões ocorreu em setembro, diminuindo com a aproximação do verão e permanecendo baixa até o início do outono. Tais resultados evidenciam que as formas ápteras de B. brassicae apresentaram menor atividade durante o verão e início do outono na região de Jaboticabal. Por outro lado, o contraste entre o número de ápteros de B. brassicae nas plantas de couve do campo 1 em relação ao campo 2 pode estar relacionado a fatores como precipitação pluvial, densidade das plantas de couve, além da sazonalidade inerente ao inseto (WelLINGS e DiXon, 1987).

Considerando-se o mesmo período de levantamento populacional nos dois campos (julho a dezembro), a precipitação pluvial foi $42,1 \%$ maior no campo 1 que no campo 2. Esse fator meteorológico apresentou correlação significativa e negativa com o número de $B$. brassicae presente no campo 1, o mesmo não se verificando no campo 2 (Quadro 4). Portanto, a maior precipitação, observada no campo 2, pode ter diminuído a densidade populacional de B. brassicae.

A densidade das plantas de couve, maior no campo 1, pode ter interferido no tamanho da população de $B$. brassicae, pois, segundo vários autores (FARREL, 1976; UvaH e CoAKER, 1984; Lal et al., 1989; HoneK e MartinKova, 1999; Karungi et al., 2000), o aumento da densidade de plantas hospedeiras pode provocar uma redução no número de pulgões, embora esta redução não seja freqüente (YAMAMURA, 1999).

Esse fenômeno tem sido atribuído à diferença visual entre plantas e a superfície exposta do solo sobre o pouso de insetos em vôo (Dent, 1991; Costello, 1995).

Com relação à distribuição de $B$. brassicae nas plantas de couve no campo 1, verificou-se que o maior número de pulgões ocorreu nas folhas medianas e o menor nas folhas apicais, e em densidade intermediária nas folhas basais. 
Analisando o levantamento populacional nos diferentes meses, observou-se que $B$. brassicae ocorreu significativa e uniformemente em maior número nas folhas medianas que nas folhas apicais e basais.

Para o campo 2 verificou-se que, a ocorrência de $B$. brassicae predominou nas folhas apicais e medianas, enquanto nas basais o número médio foi significativamente menor. Também, no conjunto dos diferentes meses, B. brassicae, ocorreu em menor número nas folhas basais, sendo esse padrão observado em praticamente todos os meses estudados. Esses dados concordam com as observações de VAN EMDEN e BASHFORD (1971), LARA et al. (1978), TRUMBLE (1982) e Ceron-hernandez e Salguero (1995), que relataram maior preferência de $B$. brassicae pelas folhas apicais de brássicas.

Com estes resultados, pode-se inferir duas causas prováveis para o diferente padrão de distribuição de B. brassicae nas posições das folhas de couve dos dois campos estudados.

Quadro 2. Número médio ( \pm EP) de formas ápteras de B. brassicae observado em diferentes folhas de couve, no campo 1 . Jaboticabal (SP), 1998/99

\begin{tabular}{lcccc}
\hline \multirow{2}{*}{ Meses } & \multicolumn{3}{c}{ Folhas } & Basal \\
\cline { 2 - 4 } & Apical & Mediana & $0,7 \pm 0,48 \mathrm{~A}$ & Média \\
\hline Julho & $0,0 \pm 0,00 \mathrm{~A}^{1}$ & $0,9 \pm 0,47 \mathrm{~A}$ & $32,2 \pm 13,95 \mathrm{~A}$ & $14,0 \pm 4,94 \mathrm{c}$ \\
Agosto & $1,6 \pm 0,63 \mathrm{~B}$ & $8,4 \pm 2,24 \mathrm{~A}$ & $42,7 \pm 7,53 \mathrm{~B}$ & $62,6 \pm 6,44 \mathrm{a}$ \\
Setembro & $47,5 \pm 12,04 \mathrm{~B}$ & $97,5 \pm 9,38 \mathrm{~A}$ & $29,6 \pm 4,85 \mathrm{~A}$ & $26,9 \pm 4,74 \mathrm{~b}$ \\
Outubro & $13,2 \pm 3,38 \mathrm{~B}$ & $38,1 \pm 12,56 \mathrm{~A}$ & $6,7 \pm 1,94 \mathrm{~B}$ & $13,9 \pm 1,93 \mathrm{c}$ \\
Novembro & $21,4 \pm 5,01 \mathrm{~A}$ & $13,6 \pm 1,28 \mathrm{~A}$ & $0,5 \pm 0,17 \mathrm{~B}$ & $1,6 \pm 0,23 \mathrm{f}$ \\
Dezembro & $1,8 \pm 0,44 \mathrm{~A}$ & $2,4 \pm 0,48 \mathrm{~A}$ & $1,2 \pm 0,38 \mathrm{~B}$ & $2,4 \pm 0,32 \mathrm{ef}$ \\
Janeiro & $1,6 \pm 0,33 \mathrm{~B}$ & $4,4 \pm 0,69 \mathrm{~A}$ & $7,1 \pm 1,40 \mathrm{~A}$ & $4,5 \pm 0,60 \mathrm{de}$ \\
Fevereiro & $1,3 \pm 0,29 \mathrm{~B}$ & $5,0 \pm 0,83 \mathrm{~A}$ & $0,7 \pm 0,27 \mathrm{~A}$ & $4,6 \pm 1,79 \mathrm{~B}$ \\
Março & $1,3 \pm 0,83 \mathrm{~A}$ & $1,1 \pm 0,31 \mathrm{~A}$ & $24,0 \pm 3,89 \mathrm{~A}$ & $4,4 \pm 0,74 \mathrm{de}$ \\
Abril & $2,6 \pm 0,81 \mathrm{~B}$ & $6,0 \pm 1,04 \mathrm{~A}$ & $23,7 \pm 4,16 \mathrm{~A}$ & $12,7 \pm 1,77 \mathrm{c}$ \\
Maio & $3,1 \pm 0,86 \mathrm{C}$ & $11,1 \pm 1,31 \mathrm{~B}$ & $4,7 \pm 2,33 \mathrm{AB}$ & $14,4 \pm 1,91 \mathrm{c}$ \\
Junho & $1,6 \pm 0,40 \mathrm{~B}$ & $17,8 \pm 1,74 \mathrm{~A}$ & $11,6 \pm 1,31 \mathrm{~B}$ & $3,3 \pm 0,93 \mathrm{f}$ \\
Julho & $0,5 \pm 0,21 \mathrm{~B}$ & $4,8 \pm 1,45 \mathrm{~A}$ & $13,0,8 \pm 0,58$ \\
\hline Média & $6,8 \pm 1,09 \mathrm{C}$ & $13,9 \pm 1,59 \mathrm{~A}$ & &
\end{tabular}

C.V. $\%=57,16$

Médias seguidas pela mesma letra, maiúsculas na horizontal e minúsculas na vertical, não diferem significativamente entre si, pelo teste de Tukey $(\mathrm{P}>0,05)$.

Quadro 3. Número médio $( \pm \mathrm{EP})$ de formas ápteras de B. brassicae observado em diferentes folhas de couve, no campo 2. Jaboticabal (SP), 1999

\begin{tabular}{lcccr}
\hline \multirow{2}{*}{ Meses } & \multicolumn{3}{c}{ Folhas } & Basal \\
\cline { 2 - 4 } & Apical & Mediana & Média \\
\hline Julho & $0,0 \pm 0,00 \mathrm{~A}^{1}$ & $0,1 \pm 0,04 \mathrm{~A}$ & $0,0 \pm 0,00 \mathrm{~A}$ & $0,1 \pm 0,02 \mathrm{~d}$ \\
Agosto & $8,9 \pm 2,01 \mathrm{~A}$ & $12,4 \pm 1,89 \mathrm{~A}$ & $2,1 \pm 1,16 \mathrm{~B}$ & $7,8 \pm 1,08 \mathrm{c}$ \\
Setembro & $146,1 \pm 19,30 \mathrm{~A}$ & $138,1 \pm 5,87 \mathrm{~A}$ & $37,4 \pm 8,95 \mathrm{~B}$ & $107,2 \pm 10,82 \mathrm{a}$ \\
Outubro & $11,3 \pm 1,73 \mathrm{~B}$ & $26,9 \pm 6,00 \mathrm{~A}$ & $7,3 \pm 1,48 \mathrm{C}$ & $15,1 \pm 1,77 \mathrm{~b}$ \\
Novembro & $5,4 \pm 1,91 \mathrm{~A}$ & $0,7 \pm 0,44 \mathrm{~B}$ & $0,1 \pm 0,07 \mathrm{~B}$ & $2,0 \pm 0,71 \mathrm{~d}$ \\
Dezembro & $12,5 \pm 5,13 \mathrm{~A}$ & $5,0 \pm 0,88 \mathrm{~A}$ & $0,1 \pm 0,03 \mathrm{~B}$ & $5,9 \pm 1,83 \mathrm{c}$ \\
\hline Média & $34,3 \pm 6,21 \mathrm{~A}$ & $34,4 \pm 5,59 \mathrm{~A}$ & $8,7 \pm 2,10 \mathrm{~B}$ & $27,6 \pm 1,70$ \\
\hline
\end{tabular}

C.V. $\%=49,07$

Médias seguidas pela mesma letra, maiúsculas na horizontal e minúsculas na vertical, não diferem significativamente entre si, pelo teste de Tukey $(P>0,05)$. 
Quadro 4. Coeficientes de correlação (r) obtidos entre o número médio de formas ápteras de B. brassicae e a média de fatores meteorológicos registrados nos sete dias anteriores a data de amostragem em couve. Jaboticabal (SP), 1998/99

\begin{tabular}{|c|c|c|c|c|c|c|c|}
\hline \multirow{2}{*}{ Local } & \multicolumn{3}{|c|}{ Temperatura } & \multirow{2}{*}{$\begin{array}{l}\text { Umidade } \\
\text { relativa }\end{array}$} & \multirow{2}{*}{ Insolação } & \multirow{2}{*}{ Precipitação ${ }^{(1)}$} & \multirow{2}{*}{$\mathrm{n}$} \\
\hline & Máxima & Mínima & Média & & & & \\
\hline & 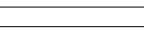 & ${ }^{\circ} \mathrm{C}-$ & 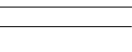 & $\%$ & horas & $\mathrm{mm}$ & \\
\hline Campo 1 & 0,06 & $-0,05$ & 0,01 & $-0,34^{*}$ & $-0,23$ & $-0,33^{*}$ & 42 \\
\hline Campo 2 & 0,14 & 0,15 & 0,10 & $-0,05$ & 0,01 & 0,03 & 17 \\
\hline
\end{tabular}

( ${ }^{1}$ Soma acumulada dos índices registrados nos sete dias anteriores a data de amostragem;

"Significativo $(\mathrm{P}<0,05) ; \mathrm{n}=$ número de pares de dados.

Primeiramente, o menor número de ápteros de B. brassicae nas folhas apicais no campo 1 pode estar relacionado à precipitação pluvial, uma vez que essas folhas apresentam uma posição mais ereta que as folhas medianas e basais, expondo mais os pulgões à ação daquele fator.

Nos meses com maior precipitação pluvial, de outubro a dezembro (43,8\% da precipitação de 1998) e de janeiro a abril (66,5\% da precipitação de 1999), ocorreu um declínio da densidade de $B$. brassicae nas folhas apicais. Porém, a menor ocorrência de $B$. brassicae nas folhas apicais em agosto e setembro de 1998, período de baixa precipitação, pode estar relacionada à irrigação por aspersão, em vez da irrigação por sulco.

Em contrapartida, a menor ocorrência de $B$. brassicae nas folhas apicais em maio, junho e julho de 1999, não encontra resposta na precipitação pluvial, uma vez que nesse período registrou-se apenas 3,3\% da precipitação do ano.

Outro fator a ser considerado é o tratamento de calcário magnesiano, efetuado somente no campo 1 , que pode ter provocado alteração na fisiologia das plantas. A menor ocorrência de B. brassicae nas folhas apicais da couve cultivada neste campo pode estar relacionada com possíveis alterações nos teores de glucosinolatos, compostos que exercem importante função na seleção da planta hospedeira para pulgões monófagos (HOPKINS et al., 1998).

De fato, B. brassicae mostra resposta positiva à presença de certas quantidades de glucosinolatos, principalmente sinigrina e alil-isotiocianato (VAN EMDEN, 1972; Cole, 1997); folhas apicais e fotossinteticamente ativas de brássicas possuem elevados teores dessas substâncias. Entretanto, verificou-se no campo 1 que a preferência alimentar do pulgão foi significativamente maior nas folhas medianas da couve (Quadro 2). Esse fato pode estar relacionado às quantidades mais adequadas de glucosinolatos presentes nestas folhas, que nas apicais e basais. Como no campo 1 realizou-se a calagem, pode ser que a aplicação do calcário magnesiano tenha proporcionado, mesmo indiretamente, alguma alteração nos teores de glucosinolatos nas folhas apicais, devido à liberação de minerais presentes no solo em função do aumento do $\mathrm{pH}$ e/ ou maior disponibilidade de cálcio e magnésio. Cubeta e Cовy (2000) verificaram a influência do cálcio e do $\mathrm{pH}$ no desenvolvimento de couve, e relataram a ocorrência de mudanças significativas no metabolismo secundário das folhas apicais.

Assim como os valores de $\mathrm{pH}$ quanto à presença de cálcio e magnésio favorecem um maior crescimento do vegetal, a aplicação de calcário magnesiano para corrigir o solo pode ter incrementado a quantidade de carboidratos das folhas adultas (medianas). Esse incremento pode estar relacionado tanto com a distribuição quanto com a concentração do cálcio, que interferem no aumento da hidrólise enzimática pelas enzimas tioglicosidases que catalizam uma das rotas metabólicas de glucosinolatos, provocando em conseqüência aumento significativo nos teores desses compostos (>100 mM) translocados no floema (Koroleva et al., 2000). Por outro lado, deve-se considerar também o efeito do magnésio, que provoca aumento significativo da atividade catalizadora das enzimas mirosinases, resultando no incremento de glucosinolatos (LUDiKHUYZE et al., 2000).

Devido à influência do cálcio e magnésio na atividade catalítica dos glucosinolatos, pode-se considerar a existência de provável correlação desses elementos na formação, quantidade e disponibilidade dos glucosinolatos, através do complexo metabolismo secundário dos vegetais.

As respostas da distribuição vertical de $B$. brassicae nas plantas de couve do campo 1, portanto, são divergentes comparando-se com as encontradas no campo 2, onde não houve tratamento de calcário magnesiano. Nesse campo, a distribuição dos pulgões ocorreu nas folhas apicais e medianas (Quadro 3), indicando que a influência do $\mathrm{pH}$ e/ou maior disponibilidade de cálcio e magnésio desse solo não tratado, pode ter tornado os teores de glucosinolatos, nessas folhas, mais adequados para a alimentação do pulgão. 
Para que essas suposições possam ser confirmadas, como sugestão para pesquisas futuras, poder-se-ia avaliar, simultaneamente, alguns parâmetros fisiológicos como a área foliar, o número de folhas, a idade foliar, os teores de nutrientes, a concentração de aminoácidos e, sobretudo, determinar e quantificar os teores de metabólitos secundários, especificamente os glucosinolatos, incluindo sinigrina e alil-isotiocianato, os quais possuem importante função na preferência alimentar, podendo interferir e modificar a distribuição vertical de B. brassicae em brássicas.

\section{CONCLUSÕES}

1. A precipitação pluvial reduz a densidade populacional de B. brassicae em plantas de couve.

2. A densidade populacional de B. brassicae é maior nas folhas medianas e apicais das plantas de couve.

\section{AGRADECIMENTOS}

Os autores agradecem ao Dr. Carlos Roberto Sousa Silva (UFSCar - São Carlos, SP) pela identificação de Brevicoryne brassicae, e ao Dr. José Carlos Barbosa (FCAV/UNESP - Jaboticabal, SP) pela orientação das análises estatísticas.

\section{REFERÊNCIAS BIBLIOGRÁFICAS}

BUENO, V.H.P.; SOUZA, B.M. Ocorrência e diversidade de insetos predadores e parasitóides na cultura de couve Brassica olerace var. acephala em Lavras, MG, Brasil. Anais da Sociedade Entomológica do Brasil, Londrina, v.22, p.5-18, 1993.

CERON-HERNANDEZ, O.J.; SALGUERO, V. Population fluctuation of aphid species (Aphididae: Hom.) and their distribution on brocoli plants (Brassica oleracea var. italica). Manejo Integrado de Plagas, Costa Rica, v.37, p.21-25, 1995.

CHURCH, B.M.; STRICKLAND, A.H. Sampling cabbage aphid populations on brussels sprouts. Plant Pathology, Oxford, v.3, p.76-80, 1954.

COLE, R.A. The relative importance of glucosinolates and amino acid to the development of two aphids pests Brevicoryne brassicae and Myzus persicae on wild and cultivated brassica species. Entomologia Experimentalis et Applicata, Dordrecht, v.85, p.121133, 1997.

COSTELLO, M.J. Spectral reflectance from a broccoli crop with vegetation or soil as background: influence on immigration by Brevicoryne brassicae and Myzus persicae. Entomologia Experimentalis et Applicata, Dordrecht, v.75, p.109-118, 1995.
CUBETA, M.A.; COBY, B. R. Influence of soil calcium, potassium, and $\mathrm{pH}$ on development of leaf tipburn of cabbage in eastern North Carolina. Communications in Soil Science and Plant Analysis, New York, v.31, n.3-4, p. 259-275, 2000.

DENT, D. Insect pest management. Wallingford: CAB International, 1991. p.373-397.

ELLIS, P.R.; SINGH, R. A review of the host plants of the cabbage aphid, Brevicoryne brassicae (Homoptera, Aphididae). IOBC/ WPRS Bulletin, v.16, p.192-201, 1993.

FARREL, J.A.K. Effects of groundnut crop density on the population dynamics of Aphis craccivora Koch (Hemiptera, Aphididae) in Malawi. Bulletin of Entomological Research, Wallingford, v.66, p.317-329, 1976.

FILGUEIRA, F.A.R. Novo manual de olericultura: agrotecnologia moderna na produção e comercialização de hortaliças.Viçosa: UFV, 2000. p.275, 279.

GEORGE, K.S. Preliminary investigations on the biology and ecology of the parasites and predators of Brevicoryne brassicae L. Bulletin of Entomological Research, Wallingford, v.48, p.619629, 1957.

HONEK, A.; MARTINKOVA, Z. Host-plant mediated influences on population development of Sitobion avenae (Sternorrhyncha: Aphididae). European Journal of Entomology, Ceske Budejovice, v.96, p.135-141, 1999.

HOPKINS, R.J.; EKBOM, B.; HENKOW, L. Glucosinolate content and susceptibility for insect attack of three populations of Sinapis alba. Journal of Chemical Ecology, New York, v.24, p.1203-1216, 1998.

KARUNGI, J.; ADIPALA, E.; OGENGA-LATIGO, M.W.; KYAMANYWA, S.; OYOBO, N. Pest management in cowpea. Part 1. Influence of planting time and plant density on cowpea field pests infestation in eastern Uganda. Crop Protection, Guildford, v.19, p.231-236, 2000.

KOROLEVA, O.A.; DAVIES, A.; DEEKEN, R.; THORPEMICHAEL, R.; TOMOS, A.D.; HEDRICH, R. Identification of a new glucosinolate-rich cell type in Arabidopsis flower stalk. Plant Physiology, v. 124, n.2, p. 599-608, 2000.

LAL, S.S.; YADAVA, C.P.; DIAS, C.A.R. Effect of planting density and chickpea cultivars on the infestations of the black aphid, Aphis craccivora Koch. Madras Agricultural Journal, Coimbatore, v.76, p.461-462, 1989.

LARA, F.M.; MAYOR JR., J.; COELHO, A.; FORNASIER, J.B. Resistência de variedades de couve a Brevicoryne brassicae (Linnaeus, 1758). I. Preferência em condições de campo e laboratório. Anais da Sociedade Entomológica do Brasil, Jaboticabal,v.7, p.175-182, 1978.

LUDIKHUYZE, L.; RODRIGO, L.; HENDRICKX, M. The activity of myrosinase from broccoli (Brassica oleracea L. cv. Italica): Influence of intrinsic and extrinsic factors. Journal of Food Protection, v.63, n.3, p.: 400-403, 2000.

OLIVEIRA, A.M. de. Observações sobre a influência de fatores climáticos nas populações de afídeos em batata. Pesquisa Agropecuária Brasileira, Brasília, v.6, p.163-172, 1971. 
PEREIRA, A.C.; SMITH, J.G. Observações sobre afídeos e seus predadores em couve-flor. Anais da Sociedade Entomológica do Brasil, Jaboticabal, v.5, p.29-33, 1976.

ROSSI, M.M.; MATIOLI, J.C.; CARVALHO, C.F. Efeitos de fatores climáticos sobre algumas espécies de pulgões (Homoptera: Aphididae) na cultura da batata, em Lavras-MG. Anais da Sociedade Entomológica do Brasil, Porto Alegre, v.19, p.75-86, 1990.

SALGADO, L.O. Pragas das brássicas, características e métodos de controle. Informe Agropecuário, Belo Horizonte, v.9, p.4347, 1983.

SOUZA, B.M. de. Efeitos de fatores climáticos e de inimigos naturais sobre Brevicoryne brassicae (Linnaeus, 1758) (Homoptera: Aphididae) em couve Brassica oleracea var. acephala (DC.) (Catparales: Brassicae). 1990. 131f. Dissertação (Mestrado em Entomologia) - Escola Superior de Agricultura de Lavras.

SOUZA, B.M. de; BUENO, V.H.P. Parasitóides e hiperparasitóides de múmias de Brevicoryne brassicae (Linnaeus, 1758) (Hemiptera: Aphididae). Revista de Agricultura, Piracicaba, v.67, p.55-62, 1992.

TRICHILO, P.J.; WILSON, L.T.; MACK, T.P. Spatial and temporal dynamics of the threecornered alfalfa hopper (Homoptera: Membracidae) on soybeans. Environmental Entomology, Lanham, v.22, p.802-809, 1993.

TRUMBLE, J.T. Within-plant distribution and sampling of aphids (Homoptera: Aphididae) on broccoli in southern California. Journal of Economic Entomology, Lanham, v.75, p.587-592, 1982.
UVAH, I.I.I.; COAKER, T.H. Effect of mixed cropping on some insect pests of carrots and onions. Entomologia Experimentalis et Applicata, Dordrecht, v.36, p.159-167, 1984.

VAN EMDEN, H.F. Aphids as phytochemists. In: HARBORNE, J.B. (Ed.). Phytochemical ecology. London: Academic Press, 1972. cap.3, p.25-43.

VAN EMDEN, H.F.; BASHFORD, M.A. The performance of Brevicoryne brassicae and Myzus persicae in relation to plant age and leaf amino acids. Entomologia Experimentalis et Applicata, Dordrecht, v.14, p.349-360, 1971.

WELLINGS, P.W.; DIXON, A.F.G. The role of weather and natural enemies in determining aphid outbreaks. In: BARBOSA, P.; SCHULTZ, J.C. (Eds.). Insect outbreaks. San Diego: Academic Press, 1987. p.313-346.

WILSON, L.T.; GUTIERREZ, A.P.; HOGG, D.B. Within-plant distribution of cabbage looper, Trichoplusia ni (Hübner) on cotton: development of a sampling plan for eggs. Environmental Entomology, Lanham, v.11, p.251-254, 1982.

WRIGHT, L.C.; CONE, W.W. Population dynamics of Brachycorynella asparagi (Homoptera: Aphididae) on undisturbed asparagus in Washington state. Environmental Entomology, Lanham, v.17, p.878-886, 1988.

YAMAMURA, K. Relation between plant density and arthropod density in cabbage fields. Researches on Population Ecology, Tokyo, v.41, p.177-182, 1999. 